\title{
Particle Physics for Primary Schools: a Case Study about Science Teaching in K-12 Schools
}

\author{
Cristina Lazzeroni, ${ }^{a}$ Sandra Malvezzi ${ }^{b}$ and Andrea Quadri ${ }^{c, d, *}$ \\ ${ }^{a}$ School of Physics and Astronomy, University of Birmingham, \\ Birmingham B15 2TT, UK \\ ${ }^{b}$ Istituto Nazionale di Fisica Nucleare, Sez. di Milano Bicocca, \\ Piazza della Scienza 3, I-20126 Milano, Italy \\ ${ }^{c}$ Istituto Nazionale di Fisica Nucleare, Sez. di Milano, Via Celoria 16, I-20133 Milano, Italy \\ ${ }^{d}$ Ministero dell'istruzione-IC E.Fermi, Carvico, \\ Via Cavour 9, I-24030 Carvico, Italy \\ E-mail: c.lazzeroni@bham.ac.uk, sandra.malvezzi@mib.infn.it, \\ andrea.quadri@mi.infn.it
}

The rapid changes in science and technology witnessed in recent decades have significantly contributed to the arousal of the awareness in decision-makers and the public as a whole of the need to strengthen the connection between academic outreach offices and educational institutions. In this context a central role should be played by schools. In the present paper we report on an ongoing project aimed to teach Particle Physics in primary schools, originally designed by C. Lazzeroni and M. Pavlidou at the University of Birmingham. The Italian version of the project was focused on the relevance of teachers' training in the adaptation of the tools and methodologies to a very different school context than the original one. We also make some general comments on several aspects related to the dissemination by research institutions of outreach materials designed for schools.

\footnotetext{
*** The European Physical Society Conference on High Energy Physics (EPS-HEP2021), ***

*** 26-30 July $2021 * * *$

*** Online conference, jointly organized by Universität Hamburg and the research center DESY ***
}

\footnotetext{
${ }^{*}$ Speaker
} 


\section{Introduction}

Disseminating recent results in modern science, and in particular in physics, as well as designing effective teaching tools in order to improve the scientific skills and competences of young children are strongly connected issues.

In today's knowledge society it has been suggested that teachers' work should be more about teaching the young generation to be aware and able to access the "multiple literacy contexts in which they live, learn and work" [1].

Science embodies conventions, tools and procedures that are an essential part of any up-to-date school curriculum. Moreover, science can be a fascinating and deeply inspiring subject for young children, who already at an early age start shaping an intuitive vision of their social role and their job once they become adults. Consequently, being aware of the relevance and wonders of contemporary science is an opportunity that schools should offer to all pupils, allowing them to fully exploit their aptitudes and skills.

Scientific knowledge is also a key component of today's citizenship. In particular, everyone should be able to appreciate the social and cultural nature of the inquiry-based learning process, with emphasis on the methodology, values and conventions of today's research in science and technology, as well as on the most up-to-date results and the commonly accepted theories confirmed by experiments.

Within this perspective the Particle Physics for Primary Schools (PPPS) project proposed by C. Lazzeroni and M. Pavlidou offers a consistent approach to the presentation of the main results of the Standard Model (SM) of Particle Physics dedicated to K-12 schools. The aim is both to offer young children an accessible yet rigorous account of the experimentally verified body of knowledge of the SM and to give a flavour of why becoming a scientist can be a passionating and wonderful career to pursue.

In this paper we give a concise account of the PPPS program, in both its original English version and Italian follow-up [2]. The latter tries to emphasize more the role of teachers as a complementary driving force together with scientists involved in outreach activities in disseminating science to young generations. It also provides an interesing example of how a science-teaching program can be applied in very different schools systems provided that different expectations and opportunities specific to each educational system are taken into account.

\section{The PPPS Program in the UK}

The original Particle Physics for Primary Schools Workshop [3-6] was developed in the UK in 2015 by C. Lazzeroni and M. Pavlidou. It was supported by a collaboration between the University of Birmingham and the Ogden Trust, with funds from a Public Engagement Fellowship (held by Lazzeroni) from the UK Science and Technology Facilities Council.

The main focus was to address the issue of creating meaningful interactions and engagement between scientists, teachers, and students at the early stages of their educational journey, with regard to modern cutting-edge science.

The early stage of the educational journey is, in fact, the most critical phase in the formation of the child's future attitude to science. The inspirational wonder of twentieth century science, and 
of Particle Physics as an example, and the message that science is a dynamic process in continuous evolution, to which children can contribute as future adults, are key factors in establishing an individual personal connection to science. The UK workshop involved a number of schools in the Birmingham area that acted as a testing ground for the first prototype. The workshop was developed first as a school or after-school or enrichment activity, to be performed in a period of about $3 \mathrm{~h}$. The workshop starts with a short introduction to the basic concepts and ideas of Particle Physics and the world of infinitesimally small scales. Particular attention is given to illustrating the main points with practical demonstrations as much as possible and to relate abstract ideas to everyday concepts to which children can relate, via metaphors and similitudes. The introduction is kept as far as possible in the format of interactive dialogue, not only as it is much more effective in terms of raising and maintaining students engagement but also because it is of paramount importance to assess the previous knowledge of the audience on the subject and tailor the content and tone accordingly. We have observed a vast difference in the children's background knowledge, with some of them already knowing about the existence of quarks and others having no idea of atoms. Although a simplification process is necessary in order to achieve an acceptable level of accessibility, a strong link to research concepts is maintained and rather sophisticated ideas such as matter-antimatter annihilation, dark matter dominance, and the various types of particle interactions and the concept of Feynman diagrams, are included in the introduction. The second part of the workshop is centered on card games, specifically designed to facilitate both learning and remembering of the concepts presented in the introduction, through playful activities. The role of enjoyment and play in early-age children's learning process is, in fact, paramount. Here the children have the chance to create mnemonic association between the particle characteristics and their behavior and interactions. The children are then divided into three groups, with each group assigned to a class of elementary particles (quarks, leptons, force mediators), and each child is asked to choose a particular elementary particle. The third part of the project aims to create a link between science and creativity, via the art-and-crafts activities of making an individual particle model of the particle of their choice, inspired by the particle characteristics and behavior, and of drawing and then producing physical models of particle interactions involving the chosen particle. In the last part of the workshop, children are re-grouped according to the particles involved in given specific, simple examples of Feynman diagrams, and are invited to illustrate the particle interaction via a storytelling process using an artistic medium of their choice: a comic strip, song, poem, or piece of creative writing or drama. Importantly, the workshop includes activities performed both as a single learner and in a group. The workshop concludes with each child presenting to the whole class the particle model and interaction models made individually and the creative work done in collaboration with others; such presentation allows verification by the researchers and teachers of the contents learned and remembered and an opportunity to improve communication skills. The workshop was delivered to a number of local schools in the UK West Midlands region, between 2015 and 2018. To evaluate the impact of the workshop, a set of questions were developed in collaboration with the teachers involved and answers were collected during, immediately after and one month after the workshop. The evaluation confirmed a strong impact in terms of increased knowledge and awareness of scientific results and methods and revealed a positive change in the attitude towards science. A set of practical resources, including learning material, lists of items for the art-and-craft activities and a comprehensive teacher manual, has been developed and used 
in teacher and educator development (CPD) courses organized by several institutions (Institute of Physics, Ogden Trust, CERN and STEM Ambassadors among others). The learning material includes a set of trump cards, which represent the known particles in the Standard Model together with their charge, mass and favorite interactions (forbidden interactions are communicated to young pupils as "dislikes", allowed interactions are instead represented by "likes").

\section{The PPPS Program in Italy}

Since 2017 the PPPS Workshop developed at the University of Birmingham has been implemented in several schools in Northern Italy. The project has been adapted to the structure of the Italian public education system. Specific attention has been paid to teacher training and on the reinforcement of the collaboration between scientists and teachers.

\subsection{The Methodology}

The Particle Physics program for primary schools relies on learning material and the wellestablished set of teaching activities designed to improve teaching and arouse the interest of the pupils, described in Section 2. A typical Particle Physics session in the primary schools at IC Carvico starts with a motivation session connecting the sub-atomic world to the macroscopic world we live in, which is of course much more familiar to young pupils. Once the notion of "atom" has been established, an inquiry-based approach is used in order to bring students to formulate the question: "What is inside the atom?". Then neutrons, protons and electrons are presented and eventually the wonderful zoo of elementary particles enters the scene. It is explored (in Italian) through the translated trump cards game, and the concrete building of particle models (colored and decorated polystyrene spheres with properties that correspond to those of the particles they represent: for instance, heavier spheres are used for heavier particles and so on). Finally, depending on the specific audience, some more advanced topics like Feynman diagrams can be introduced through the intuitive idea of storytelling, that can evolve at a later stage of the curriculum into some more mathematically precise representations of particle interactions. In 2019 the Particle Physics for Primary Schools (PPPS) project evolved into a structured training program for teachers in collaboration with INFN-Sezione di Milano Bicocca. About 90 primary school teachers in the Bergamo area took part in a two-day workshop where the basics of the Standard Model and accelerator physics, as well as an introduction to modern Cosmology, were offered in academic lectures by S. Malvezzi, C. Lazzeroni and D. Binosi (ECT*, Trento, Italy). The lectures were supplemented by demonstrations by the teachers from IC Carvico, addressed to their colleagues, on how to present in practice the material of the PPPS program to their school pupils. In addition,

an evening session dedicated to parents was organized with a lecture by S. Malvezzi. Despite the lockdown imposed by the SARS-CoV-2 pandemic, about ten of the participating schools succeeded in completing the PPPS activities in their schools, either in presence or in remote. The final results of the training program were presented in an online workshop held in November 2020.

\subsection{Results}

Several results have been achieved and are summarized as follows: 
- The interaction between professional researchers and (well-motivated) teachers is a key factor in ensuring the effectiveness of science-related teaching projects. Teachers have a huge background of competencies on how to teach in general, and an invaluable feeling of "how to teach in particular" in their classes. They can thus provide an extremely useful framework of structured programs in order to obtain a tailor-made solution for each specific class (and, ideally, each pupil). On the other hand, professional researchers play a crucial role in safeguarding the correctness of the information provided. The complexity of modern science does not lend itself to simplification or self-study. As Einstein used to say, "Everything should be made as simple as possible, but not simpler". Therefore, a thorough preliminary study of the subject under the supervision of professional researchers is a prerequisite, as well as a constant supervision of the teaching activities by the same researchers with respect to the methodology and the scientific results concerned. It should be noted that this kind of interplay is also beneficial to the transmission of the social and cultural nature of science, with its own methods and social shared values, which have to be faithfully presented to the younger generations, as has been pointed out by E.Birr-Moje in [1].

- Early exposure to science and specifically to Particle Physics enhances the appreciation of the subject and science as a whole as an interesting option to be pursued in higher education. Moreover, the question: "Which career path should I follow when I grow up?" can receive much wider and better-motivated answers in young pupils who have a feeling about what science is and how exciting it can be. This holds in particular for pupils coming from social and culturally disadvantaged backgrounds.

- Follow-up surveys filled in by pupils at the end of the project (about 50 pupils per year starting from 2017) show on average an increased motivation about studying science, with no significant gender-related bias.

\section{Distributing and Updating Science Teaching Resources}

The evolution of the digital society has changed the nature of dissemination of knowledge in a substantial way with respect to the traditional tools of letterpress printing. Digital texts can be distributed almost immediately all over the world through the WWW, taking the process of knowledge spreading, started with the introduction of press, to an unprecedented scale.

However, this is not the most important aspect of the so-called digital revolution. The big change is that nowadays everyone can become the author of digital resources, sharing them freely to potentially everyone connected to the WWW. This poses a number of additional challenges. First of all one must face the problem of trustworthy and accessible information, especially that designed to support education. Sharing information is not equivalent to allowing people to access it. One must build the competences and skills required to make such access effective.

We advocate that research agencies and universities be key players in the process of disseminating knowledge to young pupils in schools for two main reasons: firstly they can produce, review and validate the scientific soundness of the learning material; more importantly, they can redefine the paths to knowledge, deciding what comes first and what is more advanced. In this process the joint collaboration with teachers is extremely fruitful, if not essential. In the particular case 
of PPPS, particle properties and the idea of interactions through the exchange of force-carriers are what comes first and is embodied in the digital learning object of the trump cards designed at University of Birmingham. On the other hand, quantum numbers like spin or, say, the statistical properties of bosons and fermions are advanced topics, best suited to undergraduate courses.

This is an example of trustworthy and accessible information: the trump cards do contain the Standard Model of Particle Physics (and as such they are trustworthy), yet made accessible at the level of K-12 children-which means firstly establishing which information on Particle Physics is most relevant to a general audience and what is more advanced; and secondly working out the best tools, together with teachers, in order to convey such concepts by taking into account the children's psychic evolution.

\section{Conclusions}

Based on the results obtained both in the UK and the Italian programs, we conclude that the PPPS program meets some deeply rooted teaching needs. Its positive impact on students has been firmly established in both countries. The substantial difference in the school system of the two countries adds confidence that the positive results obtained are significant and could be generalized to different educational contexts. The results should be in any case verified on a larger, and ideally even more diverse, statistical sample. The pedagogical foundations of the approach also need to be further explored, in order to test whether it can be applied to a more general setting and to different physics subjects other than Particle Physics.

\section{References}

[1] Birr-Moje, E. Doing and Teaching Disciplinary Literacy with Adolescent Learners: A Social and Cultural Enterprise. Harv. Educ. Rev. 2015, 85, 254.

[2] Lazzeroni, C., Malvezzi, S., Quadri, A. Teaching Science in Today's Society: The Case of Particle Physics for Primary Schools, Universe 7 (2021) 6, 169, https: //www . mdpi . com/ 2218-1997/7/6/169.

[3] Lazzeroni, C. Particle physics for primary schools-Enthusing children to modern science. In Proceedings of the 1st Electronic Conference on Universe, online. 22-28 February 2021.

[4] Lazzeroni, C., Pavlidou, M. Particle Physics for Primary Schools-Enthusing future Physicists in Proceedings of the Conference PoS EPS-HEP2017 (2017) 564.

[5] Lazzeroni, C., Pavlidou, M. Particle Physics for Primary Schools-Enthusing future Physicists. In Proceedings of the 38th International Conference on High Energy Physics (ICHEP2016), PoS ICHEP2016 (2016) 337.

[6] Pavlidou, M., Lazzeroni, C. Particle Physics for Primary Schools-Enthusing future Physicists. Phys. Educ. 2016, 51, 054003. 